\title{
Limited specificity of commercially available SARS-CoV-2 IgG ELISAs in serum samples of African origin
}

\author{
Petra Emmerich ${ }^{1,2}$, Carolin Murawski ${ }^{3}$, Christa Ehmen ${ }^{3}$, Ronald von Possel', Neele Pekarek', \\ Lisa Oestereich ${ }^{1,4}$, Sophie Duraffour ${ }^{1,4}$, Meike Pahlmann ${ }^{1,4}$, Nicole Struck ${ }^{4,5}$, Daniel Eibach ${ }^{4,5}$, \\ Ralf Krumkamp ${ }^{4,5}$, John Amuasi ${ }^{6}$, Oumou Maiga-Ascofaré ${ }^{4,7}$, Raphael Rakotozandrindrainy ${ }^{8}$, Danny Asogun?, \\ Yemisi Ighodalo9, Simone Kann ${ }^{10}$, Jürgen May ${ }^{5}$, Egbert Tannich ${ }^{3,11}$ and Christina Deschermeier ${ }^{3}$ \\ 1 Department for Virology, Bernhard Nocht Institute for Tropical Medicine, Hamburg, Germany \\ 2 Department of Tropical Medicine and Infectious Diseases, Center of Internal Medicine II, University of Rostock, Rostock, Germany \\ 3 Department for Infectious Disease Diagnostics, Bernhard Nocht Institute for Tropical Medicine, Hamburg, Germany \\ 4 German Center for Infection Research, Hamburg - Lübeck - Borstel - Riems, Germany \\ 5 Department for Infectious Disease Epidemiology, Bernhard Nocht Institute for Tropical Medicine, Hamburg, Germany \\ 6 Global Health and Infectious Disease Research Group, Kumasi Centre for Collaborative Research in Tropical Medicine, Kumasi, \\ Ghana \\ 7 Infectious Disease Epidemiology Research Group, Kumasi Centre for Collaborative Research in Tropical Medicine, Kumasi, Ghana \\ 8 Department for Microbiology and Parasitology, University of Antananarivo, Antananarivo, Madagascar \\ 9 Institute of Lassa Fever Research and Control, Irrua Specialist Teaching Hospital, Irrua, Nigeria \\ 10 Medical Mission Institute, Würzburg, Germany \\ 11 National Reference Centre for Tropical Pathogens, Hamburg, Germany
}

Abstract

OBJECTIVES Specific serological tests are mandatory for reliable SARS-CoV-2 diagnostics and seroprevalence studies. Here, we assess the specificities of four commercially available SARSCoV-2 IgG ELISAs in serum/plasma panels originating from Africa, South America, and Europe. METHODS 882 serum/plasma samples collected from symptom-free donors before the COVID19 pandemic in three African countries (Ghana, Madagascar, Nigeria), Colombia, and Germany were analysed with three nucleocapsid-based ELISAs (Euroimmun Anti-SARS-CoV-2NCP IgG, EDI ${ }^{\mathrm{TM}}$ Novel Coronavirus COVID-19 IgG, Mikrogen recom Well SARS-CoV-2 IgG), one spike/S1-based ELISA (Euroimmun Anti-SARS-CoV-2 IgG), and in-house common cold CoV ELISAs.

RESULTS High specificity was confirmed for all SARS-CoV-2 IgG ELISAs for Madagascan (93.499.4\%), Colombian (97.8-100.0\%), and German (95.9-100.0\%) samples. In contrast, specificity was much lower for the Ghanaian and Nigerian serum panels (Ghana: NCP-based assays 77.7-89.7\%, spike/S1-based assay 94.3\%; Nigeria: NCP-based assays 39.3-82.7\%, spike/S1-based assay 90.7\%). 15 of 600 African sera were concordantly classified as positive in both the NCP-based and the spike/ S1-based Euroimmun ELISA, but did not inhibit spike/ACE2 binding in a surrogate virus neutralisation test. IgG antibodies elicited by previous infections with common cold CoVs were found in all sample panels, including those from Madagascar, Colombia, and Germany and thus do not inevitably hamper assay specificity. Nevertheless, high levels of IgG antibodies interacting with OC43 NCP were found in all 15 SARS-CoV-2 NCP/spike/S1 ELISA positive sera.

CONCLUSIONS Depending on the chosen antigen and assay protocol, SARS-CoV-2 IgG ELISA specificity may be significantly reduced in certain populations probably due to interference of immune responses to endemic pathogens like other viruses or parasites.

keywords SARS-CoV-2, seroepidemiologic studies, immunoglobulin G, Enzyme-Linked Immunosorbent Assay, specificity, Africa

Sustainable Development Goals (SDGs): Good Health and Well-Being 


\section{Introduction}

In February and early March 2020, the first COVID-19 cases were reported from African countries with a high level of international contacts (Egypt, Algeria, Nigeria, Morocco, South Africa) [1]. Following these primary importation events, case numbers rapidly increased due to intra-continental and intra-national transmission as well as further importation mainly from Europe and Asia $[1,2]$. As of February $14^{\text {th }} 2021,2,723,431$ laboratory-confirmed SARS-CoV-2 infections and 68,294 deaths caused by COVID-19 have been reported from the WHO Africa region [3].

Nevertheless, the observed incidence rates are still well below the estimated numbers that were expected based on the socioeconomic challenges many African countries are facing [4] and the numbers observed in Europe and the Americas [3]. Although testing capacities vary widely between African countries, this observation is not solely based on underreporting, but probably reflects both the lower median age of the African population and a different immune status induced by contact with endemic pathogens [4]. Besides modulating COVID-19 morbidity and mortality rates, the latter may also influence performance data of SARS-CoV-2 serological tests; in particular assay specificity may be challenged by previous or current infections with other endemic pathogens [5-7]. Therefore, analyses of this parameter in different populations are an important prerequisite to ensure reliable diagnostic procedures and seroprevalence studies.

Up to now, a plethora of ELISA tests for detection of anti-SARS-Cov-2 antibodies has been developed and commercialised [8]. Usually, these assays employ one of the major immunogenic coronavirus proteins [9] as antigen, that is the nucleocapsid protein (NCP) or defined subdomains of the spike protein (e.g. S1 or the isolated receptor binding domain (RBD)). In this study, we investigate the specificity of three NCP-based SARS-CoV-2 IgG ELISA kits and one spike/S1-based kit in pre-COVID-19 serum/plasma panels from three African countries (Ghana, Madagascar, Nigeria) as well as from South America (Colombia) and Europe (Germany).

\section{Methods}

\section{Human serum/plasma samples}

The study was performed using stored human serum/plasma samples collected before the COVID-19 pandemic (Table 1). Collection of samples was approved by

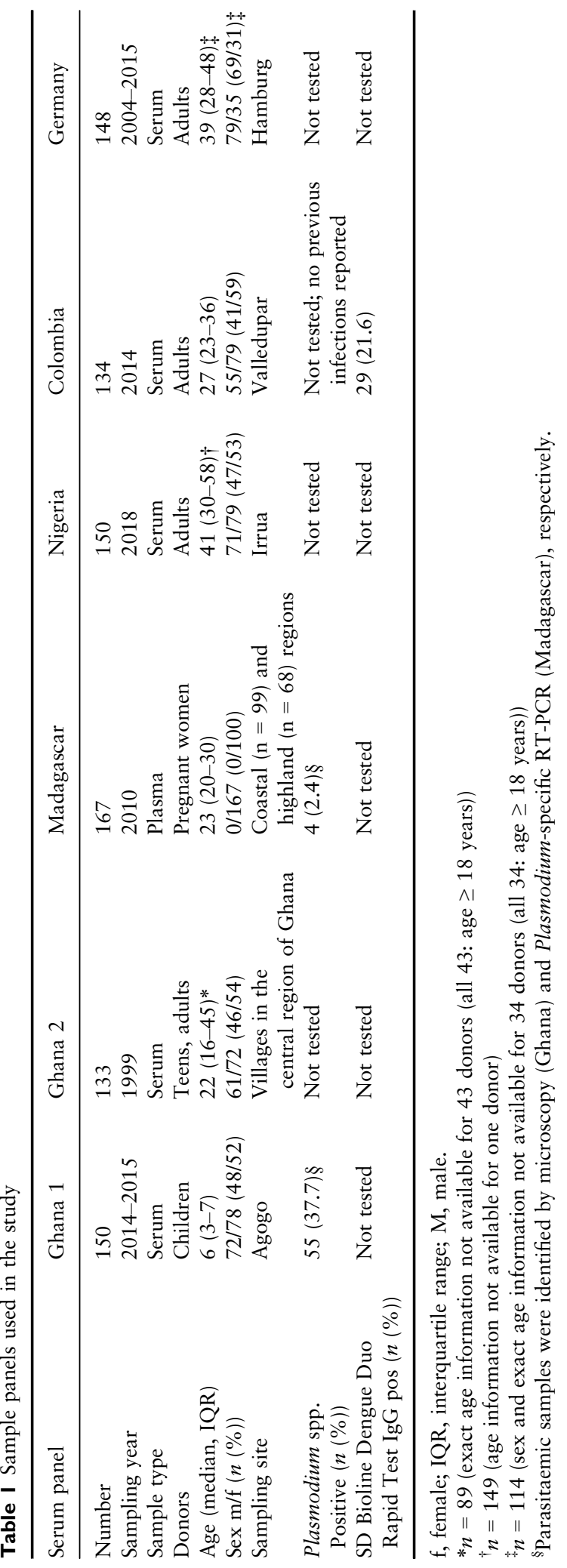


the Ethics Committees of the Kwame Nkrumah University of Science and Technology (Kumasi/Ghana; CHRPE/ AP/427/13, 2013), the Comité d'ethique de la Vice Primature Chargée de la Santé Publique (Antananarivo/ Madagaskar; no. 051-CE/MINSAN, 2009), the Irrua Specialist Teaching Hospital (Irrua/Nigeria; ISTH/HREC/ 20171019/28, 2017), the Hospital Rosario Pumarejo de Lopez (Valledupar/Colombia, 2013), and the Medical Association Hamburg/Germany (no. PV4608, 2013). All studies complied with the Declaration of Helsinki. Written informed consent was obtained from all individuals or, in case of minors, from parents or legal guardians before enrolment. Data privacy protection was guaranteed by anonymisation of samples.

\section{Commercially available assays}

SARS-CoV-2 IgG ELISAs (Euroimmun Anti-SARS-CoV2-NCP IgG ELISA (Euroimmun, Germany), Euroimmun Anti-SARS-CoV-2 IgG ELISA (Euroimmun, Germany), EDI $^{\mathrm{TM}}$ Novel Coronavirus COVID-19 IgG ELISA (Epitope Diagnostics, US), Mikrogen recomWell SARS-CoV-2 IgG ELISA (Mikrogen, Germany), Table 2), line blotting (Euroline Anti-SARS-CoV-2 Profile IgG line blot (Euroimmun)), and surrogate neutralisation assays (sVNT, Genscript, US) were performed and evaluated according to the manufacturers' instructions.

\section{SARS-CoV-2 IgG immunofluorescence testing (IIFT)}

Testing was performed as described previously [10] using SARS-CoV-2 infected Vero cells fixed with acetone/methanol.

\section{In-house common cold CoVs ELISA}

IgG antibodies were detected using a patented platform technology $[11,12]$. Briefly, diluted patient serum/plasma samples were co-incubated together with a biotinylated recombinant antigen for $24 \mathrm{~h}$ at $4{ }^{\circ} \mathrm{C}$ in a microwell plate coated with a recombinant $\operatorname{IgG}$ immune complex specific capture molecule. Following a washing step, the bound IgG/antigen immune complexes were visualised by subsequent application of horseradish peroxidase (HRP)-labelled streptavidin and the colorimetric HRP substrate tetramethylbenzidine (TMB). After stopping the enzymatic reaction, the assay result was generated by measuring the optical density of the solution in the well at 450/ $620 \mathrm{~nm}$.

As antigens, bacterially expressed, N-terminally truncated common cold $\mathrm{CoV}$ nucleoproteins comprising the protein's dimerisation domain were used (OC43 (AIL49389.1): NCP $\Delta 258$, HKU1 (AGT17773.1): NCPA256/S290F, NL63 (AFO70495.1): NCPA222, 229E (NP_073556.1): NCP $\Delta 235)$. An assay cut-off of OD450-OD620 $=0.3$ was determined by comparison of the ELISA results with the results obtained with a commercially available lineblot (Euroline Anti-SARS-CoV-2 Profile IgG, Euroimmun) for 32 German healthy blood donors. Index values (iv) were calculated by dividing the measured OD450-OD620 values by 0.3 ; samples were classified as negative (iv $<0.7$ ), borderline $(0.7 \leq$ iv $<1.3$ ), or positive (iv $\geq 1.3$ ).

Table 2 Commercially available ELISA kits used in the study

\begin{tabular}{|c|c|c|c|c|}
\hline & $\begin{array}{l}\text { Euroimmun Anti-SARS- } \\
\text { CoV-2-NCP-ELISA IgG }\end{array}$ & $\begin{array}{l}\text { Euroimmun Anti-SARS- } \\
\text { CoV-2-ELISA IgG }\end{array}$ & $\begin{array}{l}\text { EDI } I^{\mathrm{TM}} \text { Novel Coronavirus } \\
\text { COVID-19 IgG ELISA kit }\end{array}$ & $\begin{array}{l}\text { Mikrogen recom Well } \\
\text { SARS-CoV-2 IgG }\end{array}$ \\
\hline Manufacturer & $\begin{array}{l}\text { Euroimmun AG } \\
\text { Lübeck, Germany }\end{array}$ & $\begin{array}{l}\text { Euroimmun AG } \\
\text { Lübeck, Germany }\end{array}$ & $\begin{array}{l}\text { Epitope Diagnostics, Inc. } \\
\text { San Diego, US }\end{array}$ & $\begin{array}{l}\text { Mikrogen GmbH } \\
\text { Neuried, Germany }\end{array}$ \\
\hline Status & CE-IVD & CE-IVD, FDA EUA & CE-IVD & CE-IVD \\
\hline Antibody isotype & $\operatorname{IgG}$ & $\operatorname{IgG}$ & $\operatorname{IgG}$ & $\operatorname{IgG}$ \\
\hline $\begin{array}{l}\text { Test format } \\
\text { Test principle }\end{array}$ & $\begin{array}{l}96 \text { well microplate } \\
\text { indirect ELISA }\end{array}$ & $\begin{array}{l}96 \text { well microplate } \\
\text { indirect ELISA }\end{array}$ & $\begin{array}{l}96 \text { well microplate } \\
\text { indirect ELISA }\end{array}$ & $\begin{array}{l}96 \text { well microplate } \\
\text { indirect ELISA }\end{array}$ \\
\hline Antigen & NCP (modified) & spike (S1 domain) & NCP & $\mathrm{NCP}$ \\
\hline $\begin{array}{l}\text { Sample dilution } \\
\text { Interpretation } \\
\text { index value (iv) }\end{array}$ & $\begin{array}{l}\text { 1:101 } \\
\text { negative: iv }<0.8 \\
\text { borderline; } 0.8 \leq \text { iv }<1.1 \\
\text { positive: iv } \geq 1.1\end{array}$ & $\begin{array}{l}1: 101 \\
\text { negative: iv }<0.8 \\
\text { borderline; } 0.8 \leq \text { iv }<1.1 \\
\text { positive: iv } \geq 1.1\end{array}$ & $\begin{array}{l}1: 101 \\
\text { negative: iv } \leq 0.9 \\
\text { borderline; } 0.9<\text { iv }<1.1 \\
\text { positive: iv } \geq 1.1\end{array}$ & $\begin{array}{l}\text { 1:101 } \\
\text { negative: iv }<1.0 \\
\text { borderline; } 1.0 \leq \text { iv }<1.2 \\
\text { positive: iv } \geq 1.2\end{array}$ \\
\hline
\end{tabular}

CE, Conformité Européenne; EUA, Emergency Use Authorisation; FDA, Food and Drug Administration; IFU, Instructions for Use; IVD, in vitro diagnostics; NCP, nucleocapsid protein. 


\section{Statistical analysis}

Statistical analyses (calculation of $95 \%$ confidence intervals, Fisher's exact test) were performed using GraphPad Prism.

\section{Results}

High percentage of false positive SARS-CoV-2 IgG ELISA results in pre-COVID-19 serum samples from Ghana and Nigeria

To assess the specificities of commercially available SARS-CoV-2 IgG ELISA tests in sample panels of different origin, a priori SARS-CoV-2 IgG negative samples (Table 1) collected from symptom-free donors before 2019 in Africa (Ghana, Madagascar, Nigeria), South America (Colombia) and Europe (Germany) were analysed with the Euroimmun Anti-SARS-CoV-2-NCP IgG ELISA (Euroimmun, Germany), the Euroimmun AntiSARS-CoV-2 IgG ELISA (Euroimmun, Germany), the EDI $^{\mathrm{TM}}$ Novel Coronavirus COVID-19 IgG ELISA (Epitope Diagnostics, US), and the Mikrogen recomWell SARS-CoV-2 IgG ELISA (Mikrogen, Germany) (Table 2). While IgG ELISA specificities where good to excellent for pre-COVID-19 samples originating from Colombia, Madagascar, and Germany, increased false positive rates were observed in a priori SARS-CoV-2 IgG negative sera from Ghana and Nigeria (Figure 1, Table 3).

\section{Correlation of NCP- and spike/S1-based ELISA results}

The index values obtained with the three assays employing the same antigen (recombinant NCP) showed a clear correlation (Figure 2). In contrast, only a small number of 15 out of 600 African samples (Ghana 1: 4/150, Ghana 2: 3/133, Madagascar: 0/167, Nigeria: 8/150) were concordantly classified as positive by both the NCPbased and the spike/S1-based Euroimmun IgG ELISA (Figure $3 \mathrm{a}-\mathrm{c}$ ), a criterion that is fulfilled by the vast majority of sera from PCR-confirmed COVID-19 patients in the convalescent phase [13]. Positive or borderline serum reactivity with recombinant SARS-CoV-2 NCP (respectively spike/S1) was confirmed for 5/7 (7/7) Ghanaian and 6/8 (7/8) Nigerian samples using a commercially available line blot (Euroline Anti-SARS-CoV-2 Profile IgG line blot (Euroimmun)) (Figure 3d). In addition, antibodies binding to SARS-CoV-2 spike S2 domain were found in 7/7 Ghanaian and 4/8 Nigerian samples (Figure $3 \mathrm{a}-\mathrm{d})$. However, all 15 samples tested negative in IgG IIFT using SARS-CoV-2-infected Vero cells [10] and showed no or only very weak activity in a SARS-CoV-2 surrogate virus neutralising test (sVNT, Genscript, US) (Figure 3d).

\section{Assessment of antibodies directed against common cold CoVs}

To investigate the influence of previous infections with common cold CoVs on SARS-CoV-2 ELISA specificity, the complete sample panel $(n=882)$ was assayed for $\mathrm{IgG}$ antibodies interacting with the C-terminal dimerisation domains of the OC43, HKU1, NL63, and 229E NCPs using an in-house ELISA. In concordance with the worldwide occurrence of these viruses, considerable fractions of all serum panels, including the ones from Germany and Colombia, reacted positive in these tests (Figure 4).

In addition, the 15 African sera concordantly classified as positive by both the NCP-based and the spike/S1-based Euroimmun ELISA (Figure $3 \mathrm{a}-\mathrm{c}$ ) were also analysed with the Euroline Anti-SARS-CoV-2 Profile IgG line blot (Euroimmun) (Figure 3d). All of them showed a strong signal with OC43 NCP (mean index value 5.6) that by far exceeded the signals detected for the NCPs of HKU1 (mean index value 0.68), NL63 (mean index value 1.2), $229 \mathrm{E}$ (mean index value 1.0), and also SARS-CoV-2 (mean index value 1.3). Strikingly, only $2 / 7$ Ghanaian samples but $7 / 8$ Nigerian samples reacted positive in the in-house OC43 ELISA employing an N-terminally truncated OC43 NCP as antigen (Figure 3d).

\section{Influence of Plasmodium parasitaemia on ELISA specificity}

Information about Plasmodium parasitaemia was only accessible for one of the Ghanaian panels (Ghana 1, 55/ 150 samples from symptom-free children with microscopically detectable parasitaemia) and the Madagascan (4/ 167 Plasmodium-PCR positive samples) panel. Here, a reduced specificity in Ghanaian parasitaemic $v$ s. non-parasitaemic samples was observed for the Euroimmun AntiSARS-CoV-2-NCP IgG ELISA but not for the other SARS-CoV-2 IgG ELISAs (Table 4).

\section{Discussion}

In our study, we report a markedly reduced specificity of four SARS-CoV-2 IgG serological assays in serum samples originating from countries of sub-Saharan Africa, that is Ghana and Nigeria. This observation is in concordance with recent reports from Benin [7], Malawi [14], Tanzania [6], and Zambia [6] describing performance data of commercially available ELISAs [7, 14] and IIFT using eukaryotic cells overexpressing SARS-CoV-2 
P. Emmerich et al. SARS-CoV-2 ELISA specificity
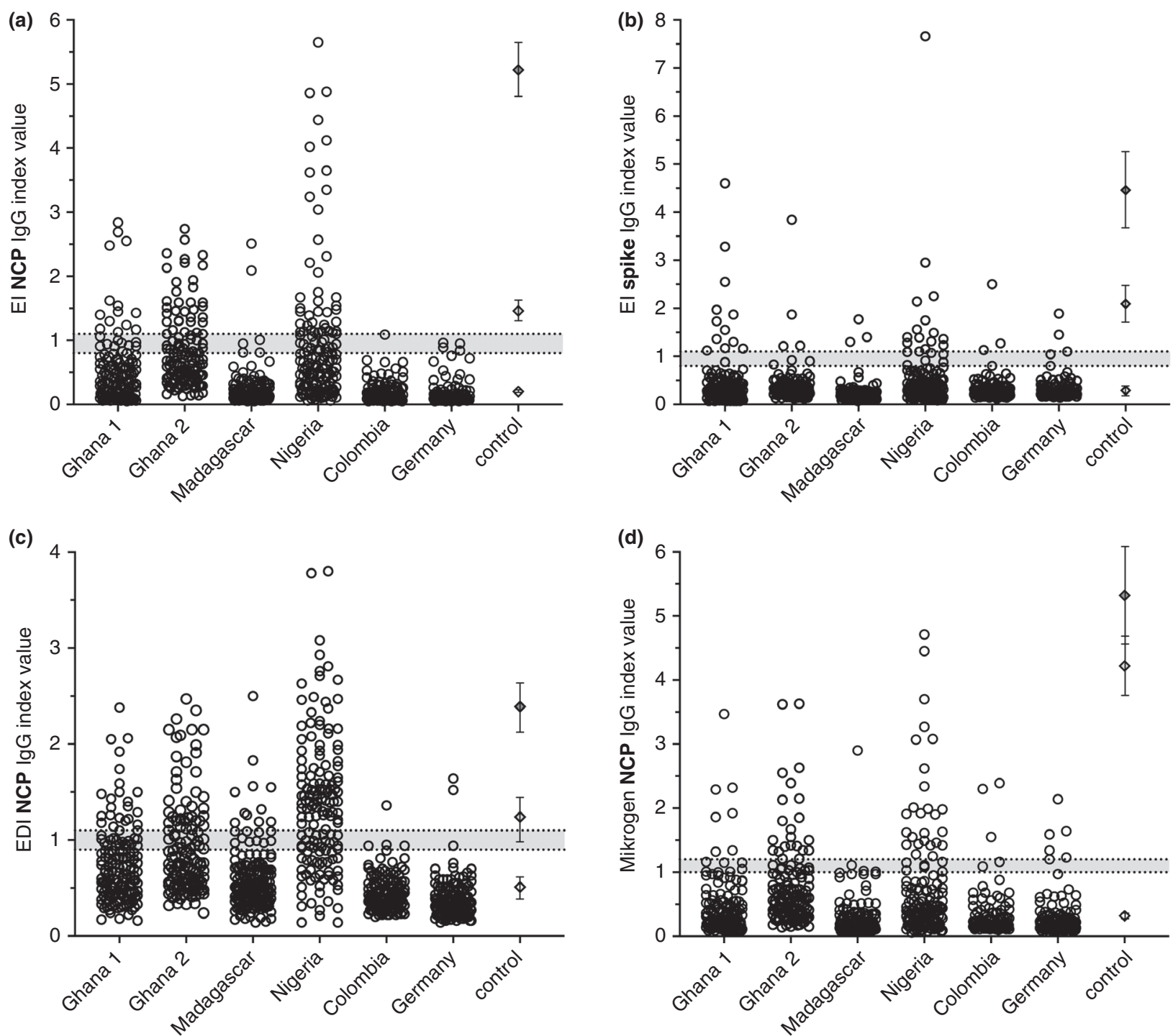

Figure I SARS-CoV-2 IgG ELISA results. (a-d) Index values obtained for serum/plasma samples collected before 2019 in three different African countries (Ghana panel $1(n=150)$, Ghana panel $2(n=133)$, Madagascar $(n=167)$, Nigeria $(n=150))$, Colombia $(n=134)$, and Germany $(n=148)$ with the Euroimmun (EI) Anti-SARS-CoV-2-NCP IgG ELISA (a), the Euroimmun (EI) Anti-SARSCoV-2 IgG ELISA (b), the EDI Novel Coronavirus COVID-19 IgG ELISA (c), and the Mikrogen recomWell SARS-CoV-2 IgG ELISA (d). Diamonds: index values obtained for two IgG positive COVID-19 patient sera sampled on day 19 post onset of symptoms (dark/light grey: SARS-CoV-2 IgG IIFT titre 1:640/1:160) and one negative control serum; error bars: standard deviation of 13 (a, c) and 14 $(\mathrm{b}, \mathrm{d})$ independent measurements. Dotted lines: negative and positive cut-off values; grey shading indicates index values rated as 'borderline' according to the manufacturers' instructions.

proteins [6], respectively. Positive reactivity with both SARS-CoV-2 NCP and spike/S1 detected by ELISA could be confirmed by line blotting for 11/15 and 14/15 African sera, respectively. Thus, the line blot is slightly less sensitive in picking up the apparently false positive signals in the pre-COVID-19 African sera. As has previously been recognised for serological tests aiming at detection of anti-SARS-CoV-1 antibodies [9], our study revealed a higher rate of false positive assay results in the NCPbased IgG ELISAs than in the spike/S1-based IgG ELISA. This finding may reflect the higher degree of sequence conservation between the immunodominant regions of 
P. Emmerich et al. SARS-CoV-2 ELISA specificity

Table 3 SARS-CoV-2 IgG ELISA specificities

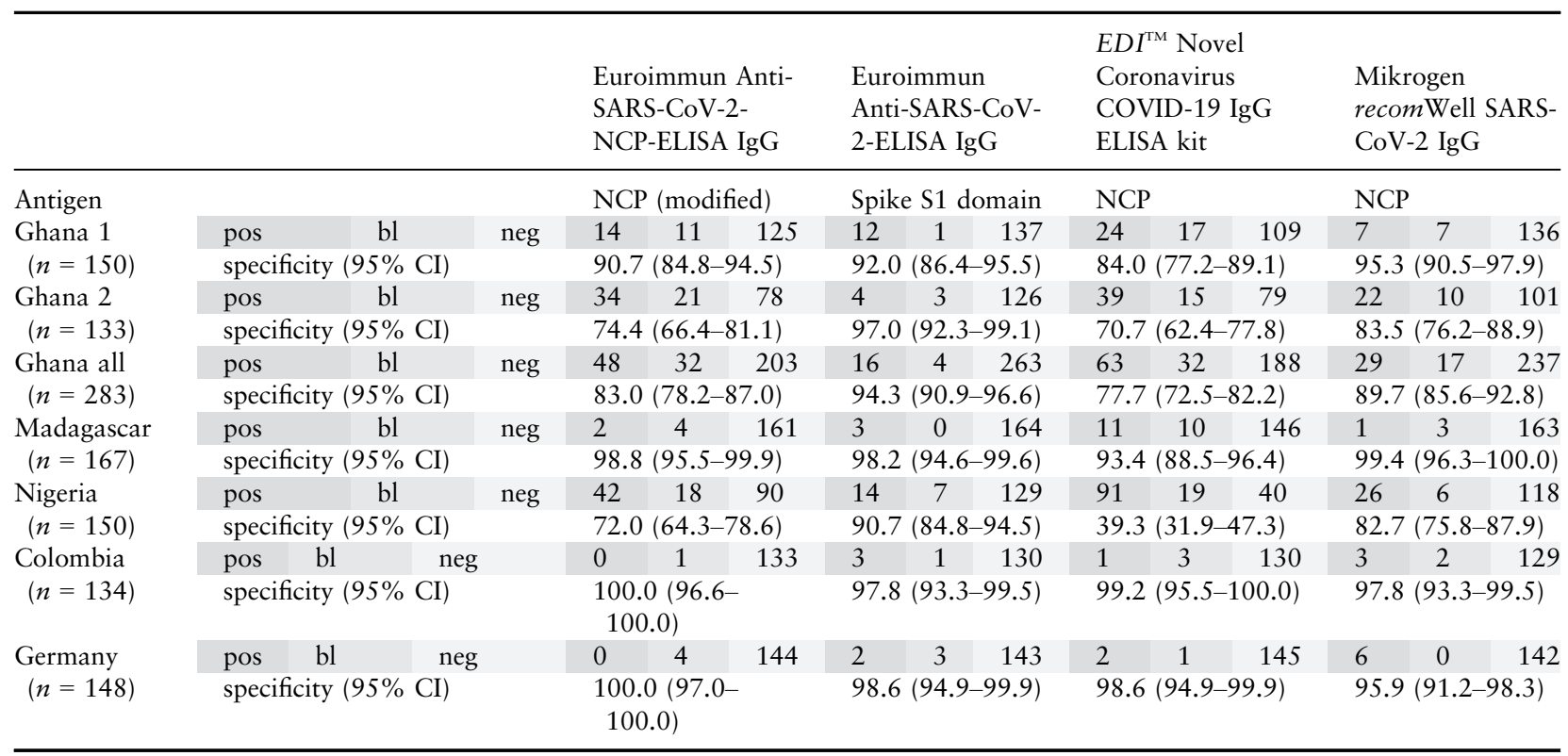

$\mathrm{CI}$, confidence interval; pos/bl/neg, number of samples rated as positive (pos), borderline (bl), and negative (neg) by the respective test. For calculation of specificities, both negative and borderline results were classified as 'not positive'.

Cells indicating the number of samples tested positive, borderline, and negative with the respective assay were shaded dark grey, medium grey, and light grey, respectively.
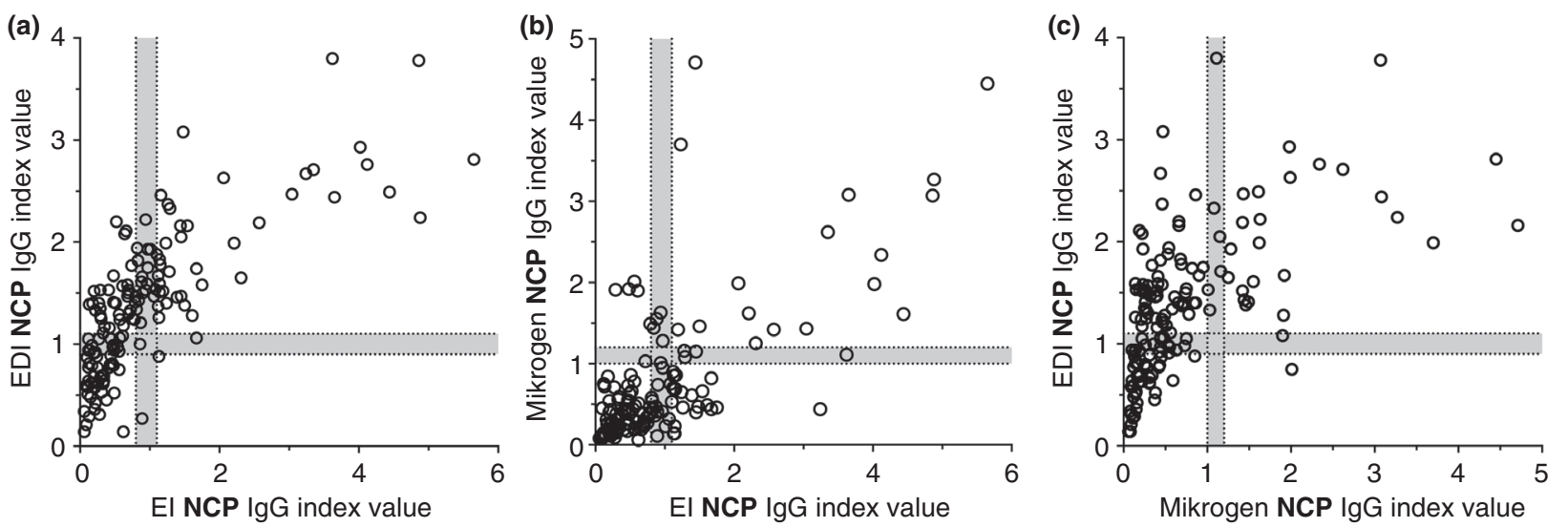

Figure 2 Correlation of SARS-CoV-2 IgG ELISA test results (NCP-based tests), exemplified for the Nigerian sample panel $(n=150)$. (a) Euroimmun (EI) Anti-SARS-CoV-2-NCP IgG ELISA vs. EDI Novel Coronavirus COVID-19 IgG ELISA, (b) Euroimmun (EI) AntiSARS-CoV-2-NCP IgG ELISA vs. Mikrogen recomWell SARS-CoV-2 IgG ELISA, (c) Mikrogen recomWell SARS-CoV-2 IgG ELISA vs. EDI Novel Coronavirus COVID-19 IgG ELISA. Dotted lines: negative and positive cut-off values; grey shading indicates index values rated as 'borderline' according to the manufacturers' instructions.

the coronavirus NCPs [9], rendering assays based on this antigen more prone to cross-reactivity than tests employing the less conserved spike protein.

Indeed, one possible cause of the observed limited specificity of SARS-CoV-2 IgG ELISAs may be cross- reactivity with antibodies elicited by previous infections with other CoVs [6]. We detected such antibodies in considerable fractions of those sample panels for which excellent SARS-CoV-2 IgG ELISA specificity has been observed. Thus, their presence does not inevitably lead to 
(a)

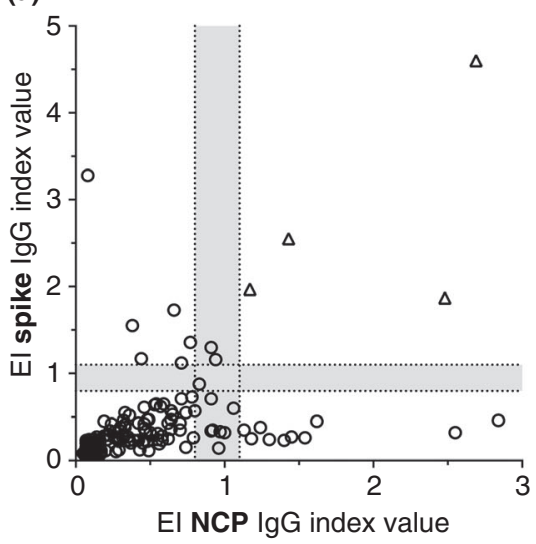

(b)

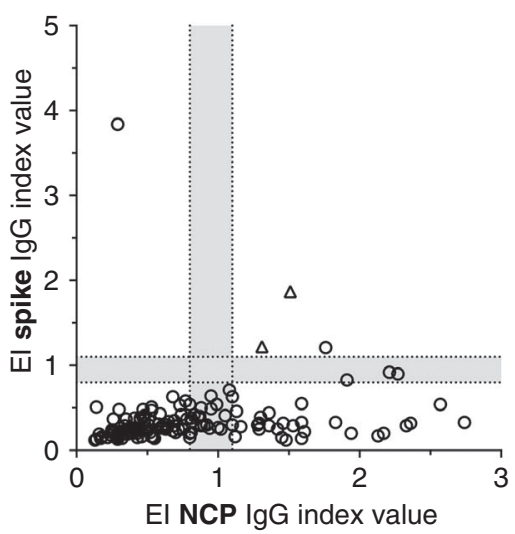

(c)

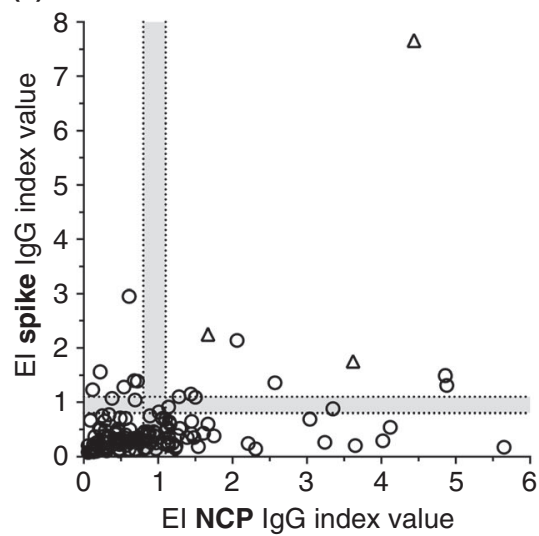

(d)

\begin{tabular}{|c|c|c|c|c|c|c|c|c|c|c|c|c|c|c|}
\hline \multirow{3}{*}{ ID } & \multirow{3}{*}{$\begin{array}{l}\text { sVNT } \\
\% \text { inh }\end{array}$} & \multirow{2}{*}{\multicolumn{2}{|c|}{$\begin{array}{c}\text { Euroimmun } \\
\text { ELISA }\end{array}$}} & \multicolumn{7}{|c|}{ Euroimmun Lineblot } & \multicolumn{4}{|c|}{ in-house ELISA } \\
\hline & & & & \multicolumn{3}{|c|}{ SARS-CoV-2 } & \multicolumn{4}{|c|}{$\mathrm{NCP}$} & \multicolumn{4}{|c|}{ NCP-CTD } \\
\hline & & $\mathrm{NCP}$ & $\mathrm{S} 1$ & $\mathrm{NCP}$ & S1 & $\mathrm{S} 2$ & OC43 & HKU1 & NL63 & $229 E$ & OC43 & HKU1 & NL63 & $229 \mathrm{E}$ \\
\hline G1-1 & 1.9 & 1.2 & 2.0 & 0.4 & 3.1 & 3.5 & 5.0 & 0.7 & 0.1 & 0.1 & 1.5 & 0.5 & 0.1 & 0.1 \\
\hline G1-2 & 9.4 & 2.7 & 4.6 & 0.9 & 5.3 & 5.2 & 6.2 & 0.5 & 0.4 & 0.2 & 0.4 & 0.3 & 0.2 & 0.2 \\
\hline G1-3 & 10.0 & 1.4 & 2.5 & 1.1 & 3.6 & 1.9 & 5.1 & 0.2 & 0.6 & 0.5 & 0.2 & 0.2 & 0.3 & 0.2 \\
\hline G1-4 & 4.1 & 2.5 & 1.9 & 0.5 & 1.6 & 2.4 & 4.1 & 0.9 & 2.8 & 1.2 & 2.9 & 0.3 & 10.1 & 0.2 \\
\hline G2-1 & 25.6 & 1.8 & 1.2 & 0.9 & 1.0 & 0.6 & 1.6 & 0.2 & 0.3 & 0.4 & 0.6 & 0.2 & 0.2 & 0.1 \\
\hline G2-2 & 24.6 & 1.5 & 1.9 & 0.9 & 1.7 & 2.2 & 7.1 & 0.4 & 2.1 & 0.9 & 0.2 & 0.3 & 2.0 & 0.1 \\
\hline G2-3 & 21.2 & 1.3 & 1.2 & 1.4 & 2.4 & 4.3 & 7.3 & 0.2 & 0.8 & 0.2 & 0.2 & 0.3 & 0.2 & 0.1 \\
\hline $\mathrm{N}-1$ & 24.4 & 3.6 & 1.8 & 3.7 & 1.3 & 1.0 & 5.3 & 0.3 & 1.0 & 2.8 & 5.3 & 0.2 & 6.0 & 0.2 \\
\hline $\mathrm{N}-2$ & 10.7 & 4.9 & 1.3 & 2.3 & 0.4 & 0.3 & 6.2 & 0.4 & 1.4 & 0.5 & 1.5 & 0.2 & 0.2 & 0.2 \\
\hline $\mathrm{N}-3$ & 23.7 & 4.4 & 7.7 & 1.1 & 6.8 & 5.6 & 7.2 & 2.7 & 1.4 & 0.8 & 7.5 & 2.8 & 0.6 & 0.3 \\
\hline $\mathrm{N}-4$ & 28.4 & 4.9 & 1.5 & 3.3 & 1.8 & 0.8 & 4.1 & 1.0 & 3.0 & 2.2 & 1.4 & 0.6 & 7.4 & 0.3 \\
\hline $\mathrm{N}-5$ & 19.4 & 2.6 & 1.4 & 0.3 & 0.7 & 0.2 & 5.8 & 0.5 & 1.1 & 0.7 & 1.5 & 0.4 & 0.3 & 0.2 \\
\hline $\mathrm{N}-6$ & 21.5 & 2.1 & 2.1 & 1.2 & 1.6 & 0.2 & 4.2 & 0.3 & 0.7 & 3.5 & 4.3 & 0.9 & 1.1 & 2.6 \\
\hline $\mathrm{N}-7$ & 27.7 & 1.7 & 2.2 & 1.3 & 2.2 & 2.3 & 5.1 & 0.9 & 1.5 & 0.7 & 7.4 & 2.9 & 9.8 & 2.0 \\
\hline $\mathrm{N}-8$ & 28.3 & 1.4 & 1.2 & 0.4 & 1.0 & 0.3 & 5.0 & 1.0 & 1.2 & 0.7 & 1.0 & 0.7 & 0.9 & 0.3 \\
\hline
\end{tabular}

Figure $3(\mathrm{a}-\mathrm{c})$ Correlation of Euroimmun Anti-SARS-CoV-2-NCP IgG ELISA and Euroimmun Anti-SARS-CoV-2 IgG ELISA test results (NCP-based vs. spike/S1-based test). Shown are index values for sample panels Ghana 1 (a), Ghana 2 (b), and Nigeria (c). Triangles indicate spike/S1/NCP IgG positive samples containing IgG antibodies reacting positive with SARS-CoV-2 spike/S2 in the Euroimmun line blot. (D) ELISA, sVNT and line blot results for sera testing positive in both the Euroimmun NCP-and spike/S1-based IgG ELISA. Serum samples (Ghana $1(\mathrm{G} 1): n=4$, Ghana $2(\mathrm{G} 2): n=3$, Nigeria (N): $n=8)$ ) were tested using the SARS-CoV-2 sVNT (Genscript) and the Euroline Anti-SARS-CoV-2 Profile IgG (Euroimmun) according to the manufacturer's instructions. Rating of index values (iv): Euroimmun ELISA: negative: iv $<0.8$, borderline; $0.8 \leq$ iv $<1.1$, positive: iv $\geq 1.1$; Line blot: negative: iv $<0.6$, borderline: $0.6 \leq$ iv $<1.0$, positive: iv $\geq 1.0$; in-house ELISA: negative: iv $<0.7$, borderline: $0.7 \leq$ iv $<1.3$, positive: iv $\geq 1.3$; sVNT: negative: $\%$ inhibition $<20.0$, borderline; $20.0 \leq \%$ inh $<30.0$, positive: $\%$ inh $\geq 30.0$; CTD: C-terminal domain; dark grey fields: positive; light grey fields: borderline.

false positive signals. Nevertheless, the most challenging sample panel originating from Nigerian donors displayed the by far highest percentage of OC43 in-house-ELISA reactive samples $(43.3 \%)$ and all 15 African samples (8 from Nigeria, 7 from Ghana) showing up false positive in both NCP- and spike/S1-based assays show extraordinarily high signals in an OC43 NCP line blot. Strikingly, all 8 Nigerian samples, but only 2/7 Ghanaian samples reacted with an $\mathrm{N}$-terminally truncated, recombinant
OC43 NCP in the in-house ELISA. This discrepancy between the line blot and the in-house ELISA results may be caused by (i) a lower sensitivity of the ELISA employing a truncated NCP displaying fewer epitopes as antigen (although an ELISA based on similar fragment of SARS-CoV-1 has been shown to detect antiSARS-CoV-1 antibodies with excellent sensitivity [15]), (ii) a different conformational status of the used antigens (line blot: at least partially denatured, ELISA: 
P. Emmerich et al. SARS-CoV-2 ELISA specificity

(a) $\quad 0$ 43

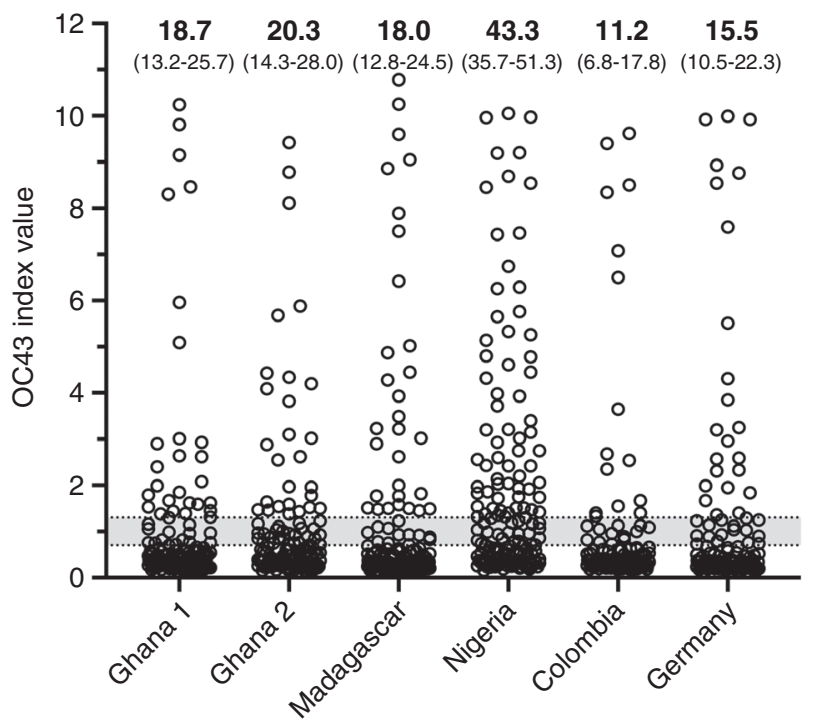

(c) NL63

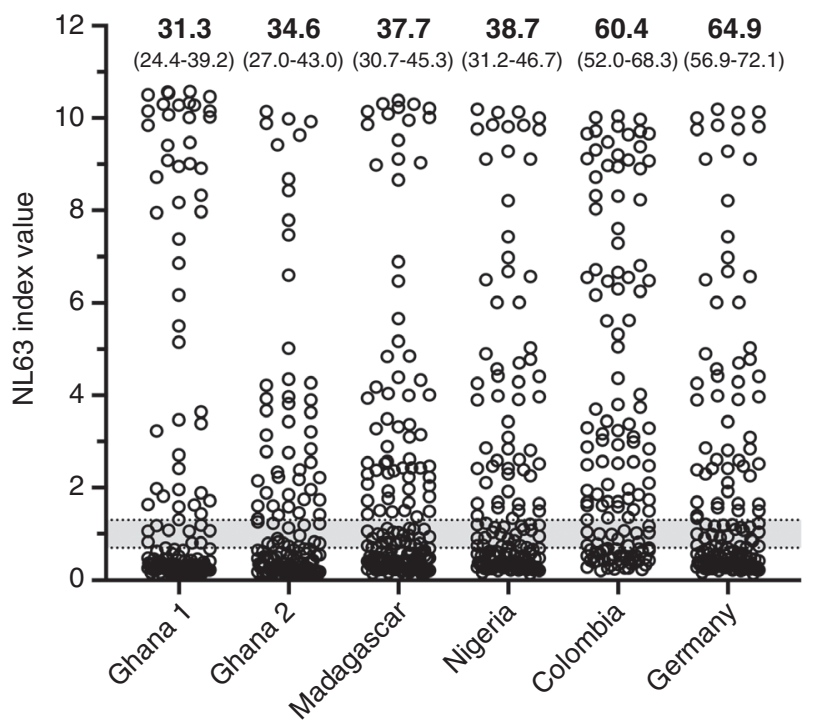

(b) HKU1

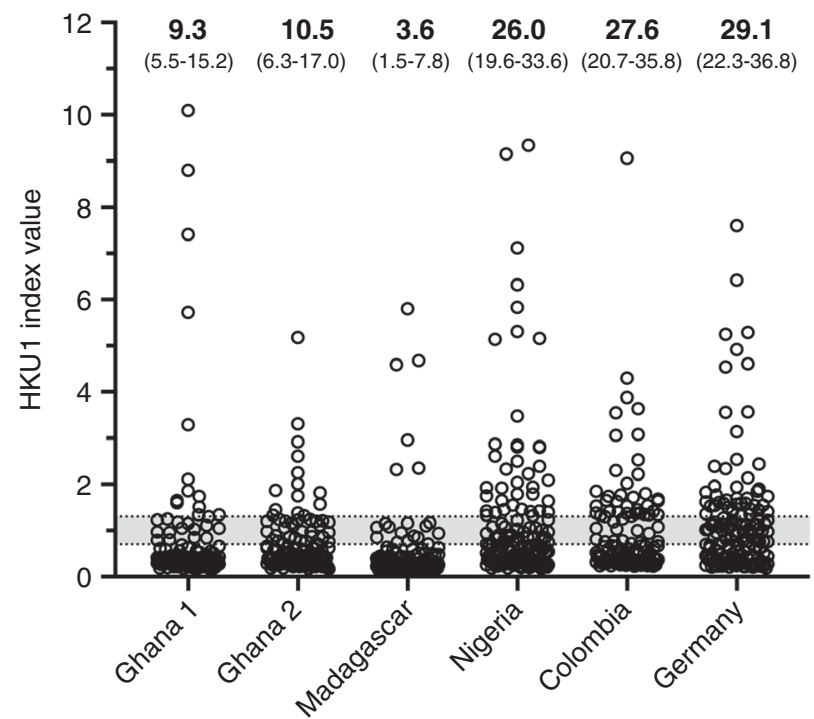

(d) 229E

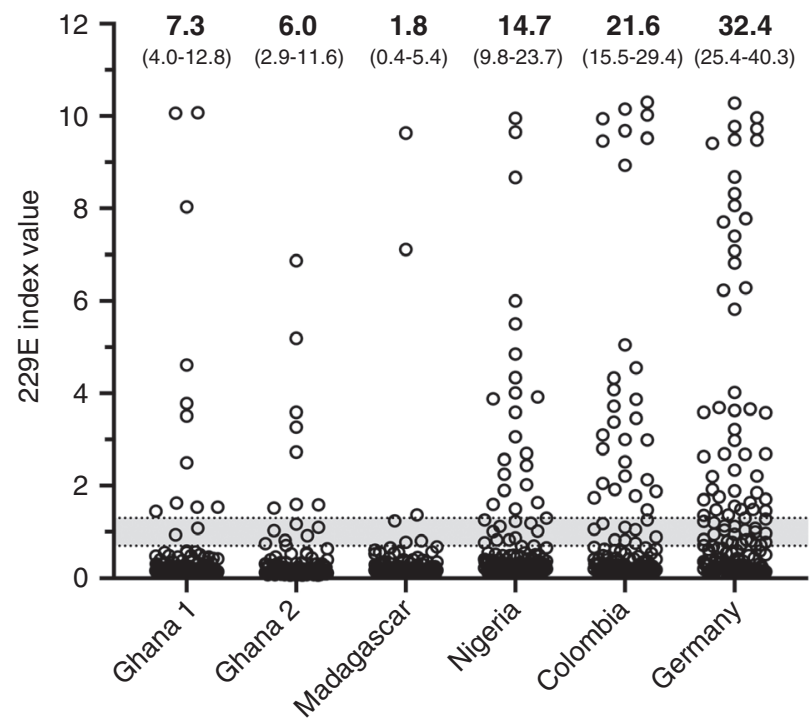

Figure 4 Common cold CoVs ELISA results. Index values obtained for the sample panels from Ghana (1: $n=150,2: n=133)$, Madagascar $(n=167)$, Nigeria $(n=150)$, Colombia $(n=134)$, and Germany $(n=148)$ using an in-house IgG ELISA protocol employing the C-terminal dimerisation domain of (a) OC43 NCP, (b) HKU1 NCP, (c) NL63 NCP, and (d) 229E NCP as antigen. Bold numbers: $\%$ of samples for which an iv $\geq 1.3$ was obtained, numbers in brackets: $95 \%$ confidence interval. Grey shading indicates ivs rated as 'borderline' $(0.7 \leq$ iv $<1.3)$.

natively folded, soluble protein), (iii) blockage of epitopes by biotinylation of the ELISA antigen, (iv) different OC43 genotypes or closely related CoVs eliciting cross-reactive immune responses in the Nigerian and Ghanaian samples, respectively (this assumption is supported by the finding that $6 / 7$ Ghanaian samples but only 4/8 Nigerian samples react borderline or positive with SARS-CoV-2 spike/S2 in the line blot). Taken together, at least some of the observed false positive signals could be due to previous infections with other coronaviruses sharing B cell epitopes with both OC43 and SARS-CoV-2. 
P. Emmerich et al. SARS-CoV-2 ELISA specificity

Table 4 SARS-CoV-2 IgG ELISA specificities for donor subgroups from Ghana with and without Plasmodium parasitaemia (Ghana panel 1 , symptom-free children)

\begin{tabular}{|c|c|c|c|c|c|c|c|c|c|c|c|}
\hline \multirow{3}{*}{$\begin{array}{l}\text { Parasitaemic } \\
(n=55)\end{array}$} & \multirow{3}{*}{$\begin{array}{l}\text { pos bl neg } \\
\text { specificity }(95 \% \mathrm{CI})\end{array}$} & \multicolumn{2}{|c|}{$\begin{array}{l}\text { Euroimmun } \\
\text { Anti-SARS-CoV- } \\
\text { 2-NCP-ELISA } \\
\text { IgG }\end{array}$} & \multicolumn{2}{|c|}{$\begin{array}{l}\text { Euroimmun } \\
\text { Anti-SARS-CoV- } \\
\text { 2-ELISA IgG }\end{array}$} & \multicolumn{3}{|c|}{$\begin{array}{l}\text { EDI }{ }^{\mathrm{TM}} \text { Novel } \\
\text { Coronavirus } \\
\text { COVID-19 IgG } \\
\text { ELISA kit }\end{array}$} & \multicolumn{3}{|c|}{$\begin{array}{l}\text { Mikrogen } \\
\text { recomWell } \\
\text { SARS-CoV-2 } \\
\text { IgsG }\end{array}$} \\
\hline & & \multirow{2}{*}{\multicolumn{2}{|c|}{$\begin{array}{lcr}9 & 6 & 40 \\
83.6 & (71.5-91.4)\end{array}$}} & $\begin{array}{ll}6 & 1\end{array}$ & 48 & 12 & 9 & 34 & 1 & 4 & 50 \\
\hline & & & & \multicolumn{2}{|c|}{$89.1(77.8-95.3)$} & \multicolumn{3}{|c|}{$78.2(65.5-87.2)$} & \multicolumn{3}{|c|}{$\begin{array}{c}98.2(89.5- \\
100.0)\end{array}$} \\
\hline \multirow{2}{*}{$\begin{array}{l}\text { Not } \\
\text { parasitaemic } \\
(n=95)\end{array}$} & pos & 5 & 85 & 6 & 89 & 12 & 8 & 75 & 6 & 3 & 86 \\
\hline & specificity $(95 \% \mathrm{CI})$ & \multicolumn{2}{|c|}{$94.7(88.0-98.0)$} & \multicolumn{2}{|c|}{$93.7(86.6-97.3)$} & \multicolumn{3}{|c|}{$87.4(79.1-92.8)$} & \multicolumn{3}{|c|}{93.7 (86.6-97.3) } \\
\hline \multicolumn{2}{|l|}{$P$ value } & \multicolumn{2}{|l|}{0.0387} & \multicolumn{2}{|l|}{0.3578} & \multicolumn{3}{|c|}{0.1675} & \multicolumn{3}{|c|}{0.4235} \\
\hline
\end{tabular}

pos/bl/neg: number of samples rated as positive (pos), borderline (bl), and negative (neg) by the respective test. CI: confidence interval. For calculation of specificities, both negative and borderline results were classified as 'not positive'. P values were calculated using Fisher's exact test $(2 \times 2$ contingency table, pos versus 'not positive').

Cells indicating the number of samples tested positive, borderline, and negative with the respective assay were shaded dark grey, medium grey, and light grey, respectively.

As has already been reported for ZIKV [16] and SARSCoV-2 IgG ELISAs [7], hypergammaglobulinaemia resulting from polyclonal B-cell activation induced by pathogens like Plasmodia can challenge assay specificity. Indeed, we observed a slightly reduced specificity of the Euroimmun Anti-SARS-CoV-2-NCP IgG ELISA in Ghanaian parasitaemic $v$ s. non-parasitaemic samples (83.6\% (95\% CI: $71.5 \%-91.4 \%$ ) vs. $94.7 \%$ (95\% CI: $88.0 \%-98.0 \%), P=0.0387)$ ). While Plasmodium falciparum malaria is holoendemic in many regions in Ghana and Nigeria [17], length and intensity of malaria transmission varies significantly between different areas in Madagascar [17, 18]. In Colombia, $78 \%$ of the population lives in malaria free areas, and Plasmodium falciparum accounts only for $58 \%$ of malaria cases $(42 \%$ : Plasmodium vivax)[19]. None of the 134 Colombian donors included in our study reported a previous malaria episode.

Recently, Lustig et al. [5] observed false positive results of spike-based IgA and IgG ELISAs in sera from donors with acute or past dengue virus infection. In the context of the study for which the samples were originally collected, $29(21.6 \%)$ of the 134 Colombian donors had been tested positive in the SD Bioline Dengue Duo IgG Rapid Test (Alere/Abbott, USA), indicating a high titre of anti-DENV IgG antibodies [20]. Indeed, two of the three Colombian sera tested positive in the spike/S1-based Euroimmun Anti-SARS-CoV-2 ELISA belonged to this subgroup; a robust statistical evaluation of this finding would require significantly higher sample numbers.

Although antibodies binding to all three recombinant SARS-CoV-2 antigens presented on the Euroline Anti-
SARS-CoV-2 Profile IgG line blot (NCP, spike/S1, spike/ S2) were detected in some pre-COVID-19 African sera generating false positive ELISA results (Figure 3d), none of these samples showed up false positive in the in-house IgG IIFT using SARS-CoV-2 infected Vero cells. A possible explanation for this surprising observation might be the use of full virus displaying native proteins in their natural context vs. highly concentrated, purified, recombinant antigens. Indeed, increased numbers of false positive SARS-CoV-2 IgG IIFT results have been observed in African sera in a study using transfected HEK cells overexpressing SARS-CoV-2 antigens [6]. Furthermore, the specific SARS-CoV-2 staining pattern might be only generated when antibodies recognising different viral proteins with sufficiently high affinity are present in a tested serum. Interestingly, line blot intensity patterns for the different SARS-CoV-2 proteins (NCP, spike/S1, spike/S2) vary strongly between the 15 sera generating false positive signals in both the NCP and Spike IgG ELISA (Figure $3 \mathrm{~d})$.

\section{Limitations of the study}

Our study has some limitations that have to be acknowledged when interpreting the presented data. First of all, the sample panels (originating from previous studies with different scientific objectives) do not reflect representative cross-sections of the respective countries' populations and also comparability between sample panels is limited. Although in total 600 African samples have been analysed, the number of samples per country giving rise to false positive SARS-CoV-2 IgG assay results is still 
relatively small, impeding statistical analyses. Furthermore, limited accessible sample volumes prevented us from performing material-intensive assays as the SD Bioline Dengue Duo IgG Rapid Test or Plasmodium-specific RT-PCR for the complete panel.

\section{Conclusions}

Our work shows that several commercially available SARS-CoV-2 IgG ELISAs, especially those employing recombinant NCP as antigen, are prone to generate a high number of false positive results when testing serum/plasma samples originating from Sub-Saharan Africa. Beside other factors, high antibody titres resulting from previous infections with other coronaviruses and/or acute or previous malaria episodes may cause this phenomenon.

Based on these findings, the following recommendations should be considered when testing sera from African individuals or performing SARS-CoV-2 seroprevalence studies (not only) in Africa: (i) Carefully assess false positive signals obtained with the chosen serological test(s) in the target population (using a priori SARS-CoV-2 IgG negative serum samples which were stocked before 2019); if necessary adjust assay cut-off [14]. (ii) Be aware of potentially interfering/cross-reacting endemic pathogens and carefully interpret SARS-CoV-2 IgG test results in this context. (iii) If possible, combine information from two independent serological tests employing different antigens. In the context of the upcoming implementation of SARS-CoV-2 vaccine programmes, even other antigens than NCP and spike, for example ORF8 and ORF3b [21], might become relevant for differentiation of natural infections from vaccine responses although their use is limited due to high genetic variability [22, 23]. (iv) Re-evaluate samples generating a positive ELISA result by SARS-CoV-2 IgG IIFT and SARS-CoV-2 neutralisation testing.

\section{Acknowledgements}

The authors thank M. Panning for providing common cold coronavirus RNAs. J. Hansen is thanked for expert technical assistance. The study was supported by the German Federal Ministry of Education and Research (Grant no. 01KI20210), the German Research Foundation (DFG) (GU 883/4-1 and GU 883/5-1), by the German Federal Ministry of Health through support of the WHO Collaborating Centre for Arboviruses and Hemorrhagic Fever Viruses at BNITM (agreement ZMV I12517WHO005), through the Global Health Protection
Program (agreement ZMV I1-2517GHP-704), and through the COVID support agreement ZMVI12520COR001. Collection of Columbian serum samples was supported by the European Regional Development Fund (ERDF), project number BWF/H/52228/2012/ 13.10.10-1/3.4. The funders had no role in study design, data collection and analysis, decision to publish, or preparation of the manuscript. Open Access funding enabled and organized by Projekt DEAL.

\section{References}

1. Studies ACfS. https://africacenter.org/spotlight/mapping-riskfactors-spread-covid-19-africa/. 2020.

2. Skrip L, Selvaraj P, Hagedorn B et al. Seeding COVID-19 across sub-Saharan Africa: an analysis of reported importation events across 48 countries. 2020. https://doi.org/10. 1101/2020.04.01.20050203

3. WHO. COVID-19 Weekly Epidemiological Update, 16 Feb 2021.

4. Mbow M, Lell B, Jochems SP et al. COVID-19 in Africa: Dampening the storm? Science 2020: 369: 624-626.

5. Lustig Y, Keler S, Kolodny R et al. Potential antigenic crossreactivity between SARS-CoV-2 and Dengue viruses. Clin Infect Dis. 2020. https://doi.org/10.1093/cid/ciaa1207

6. Tso FY, Lidenge SJ, Pena PB et al. High prevalence of preexisting serological cross-reactivity against severe acute respiratory syndrome coronavirus-2 (SARS-CoV-2) in sub-Saharan Africa. Int J Infect Dis. 2021: 102: 577-583.

7. Yadouleton A, Sander AL, Moreira-Soto A et al. Limited specificity of serologic tests for SARS-CoV-2 antibody detection, Benin. Emerg Infect Dis. 2021: 27: 233-237.

8. University JH. https://www.centerforhealthsecurity.org/re sources/COVID-19/serology/Serology-based-tests-forCOVID-19.html. 2020.

9. Meyer B, Drosten C, Muller MA. Serological assays for emerging coronaviruses: challenges and pitfalls. Virus Res. 2014: 194: 175-183.

10. Reisinger EC, von Possel R, Warnke P et al. Screening of mothers in a COVID-19 low-prevalence region: determination of SARS-CoV-2 antibodies in 401 mothers from Rostock by ELISA and confirmation by immunofluorescence. Dtsch Med Wochenschr. 2020: 145(17): e96-e100.

11. Emmerich P, Schmitz H. Patent EP2492689. 2011.

12. Schmitz H, Gabriel M, Emmerich P. Specific detection of antibodies to different flaviviruses using a new immune complex ELISA. Med Microbiol Immunol. 2011: 200: 233-239.

13. Van Elslande J, Decru B, Jonckheere S et al. Antibody response against SARS-CoV-2 spike protein and nucleoprotein evaluated by four automated immunoassays and three ELISAs. Clin Microbiol Infect 2020: 26: 1557.e1-1557.e7.

14. Chibwana MG, Jere KC, Kamn'gona R, et al. High SARSCoV-2 seroprevalence in health care workers but relatively low numbers of deaths in urban Malawi. medRxiv preprint 2020. https://doi.org/10.1101/2020.07.30.20164970 
P. Emmerich et al. SARS-CoV-2 ELISA specificity

15. Yu F, Le MQ, Inoue $\mathrm{S}$ et al. Evaluation of inapparent nosocomial severe acute respiratory syndrome coronavirus infection in Vietnam by use of highly specific recombinant truncated nucleocapsid protein-based enzyme-linked immunosorbent assay. Clin Diagn Lab Immunol. 2005: 12: 848-854.

16. Van Esbroeck M, Meersman K, Michiels J, Arien KK, Van den Bossche D. Letter to the editor: Specificity of Zika virus ELISA: interference with malaria. Euro Surveillance 2016:

21. https://doi.org/10.2807/1560-7917.ES.2016.21.21.30237

17. Gething PW, Patil AP, Smith DL et al. A new world malaria map: plasmodium falciparum endemicity in 2010. Malar J. 2011: 10: 378 .

18. Maiga-Ascofare O, Rakotozandrindrainy R, Girmann M et al. Molecular epidemiology and seroprevalence in asymptomatic Plasmodium falciparum infections of Malagasy pregnant women in the highlands. Malar J. 2015: 14: 188.
19. WHO. Malaria profile Colombia.

20. Blessmann J, Winkelmann Y, Keoviengkhone L et al. Assessment of diagnostic and analytic performance of the SD Bioline Dengue Duo test for dengue virus (DENV) infections in an endemic area (Savannakhet province, Lao People's Democratic Republic). PLoS One 2020: 15: e0230337.

21. Hachim A, Kavian N, Cohen CA et al. ORF8 and ORF3b antibodies are accurate serological markers of early and late SARS-CoV-2 infection. Nat Immunol. 2020: 21: 12931301.

22. Lam JY, Yuen CK, Ip JD et al. Loss of orf3b in the circulating SARS-CoV-2 strains. Emerg Microbes Infect. 2020: 9: 2685-2696.

23. Pereira F. SARS-CoV-2 variants lacking a functional ORF8 may reduce accuracy of serological testing. J Immunol Methods. 2021: 488: 112906.

Corresponding Author Christina Deschermeier, Bernhard Nocht Institute for Tropical Medicine, Bernhard-Nocht-Str. 74, 20359 Hamburg, Germany. Tel.: +49 4042818 438; Fax +49 4042818 400; E-mail: descherm@bnitm.de 